\title{
On the Relation between One-Species Diffusion-Limited Coalescence and Annihilation in One Dimension
}

\author{
Daniel ben-Avraham ${ }^{1}$ and Éric Brunet ${ }^{2}$ \\ ${ }^{1}$ Department of Physics, Clarkson University, Potsdam NY 13699-5820, USA \\ ${ }^{2}$ École Normale Supérieure, 24 rue Lhomond, 75230 Paris Cedex 05, France
}

\begin{abstract}
The close similarity between the hierarchies of multiple-point correlation functions for the diffusion-limited coalescence and annihilation processes has caused some recent confusion, raising doubts as to whether such hierarchies uniquely determine an infinite particle system. We elucidate the precise relations between the two processes, arriving at the conclusion that the hierarchy of correlation functions does provide a complete representation of a particle system on the line. We also introduce a new hierarchy of probability density functions, for finding particles at specified locations and none in between. This hierarchy is computable for coalescence, through the method of empty intervals, and is naturally suited for questions concerning the ordering of particles on the line.

PACS numbers: 02.50.-r, 05.40.-a, 05.70.Ln, 82.20.-w
\end{abstract}

Diffusion-limited coalescence, $A+A \rightarrow A$, and annihilation, $A+A \rightarrow 0$, on the line, have long been known to display anomalous kinetics (different from the mean-field reaction-limited regime) and to belong to the same universality class [1]- 31]. In fact, the similarities run deeper than that, as the full hierarchy of multiple-point correlation functions in the two processes, when expressed in different length scales, are identical 18, 19, 20, 21, 22]. Yet there exist important differences between the two processes. Most conspicuously, the density function for the gap $x$ between adjacent particles (the so called inter-particle distribution function, or IPDF) falls off, for $x \rightarrow \infty$, as $e^{-\alpha x^{2}}$ for coalescence, but only as $e^{-\beta x}$ for annihilation [1, 23, 26, 31]. This raises the question whether the infinite hierarchy of multiple-point correlation functions uniquely determines a system of particles on the line [22].

In this communication, we answer this question on the affirmative: the hierarchy of multiple-point correlation functions does provide a unique representation of an infinite particle system. Indeed, the IPDF can be computed from the multiple-point correlation functions using an inclusion-exclusion formula, as shown in Eq. (3). The correlation functions of the two processes are simply not the same, despite the similarity upon the rescaling of space. The precise relationship between coalescence and annihilation, and its consequences, is thoroughly discussed. An alternative hierarchy of probability density functions, for finding particles at locations $x_{1}, \ldots, x_{n}$ and none in between, is introduced, and we show how to compute it explicitly, for coalescence, using the method of empty intervals 26, 27, 28, 29, 30, 32]. This new hierarchy is naturally suited for answering questions concerning the ordering of particles on the line. It is further used for obtaining an exact new expression, as well a series of systematic approximations for the IPDF of the annihilation process [23, 31].

\section{Equivalence between coalescence and annihilation}

The $n$-point correlation functions (the joint probability for finding particles at positions $x_{1}, \ldots, x_{n}$ at time $t$, simultaneously) of diffusion-limited annihilation and diffusion-limited coalescence in one dimension seem very similar. Indeed, for suitable initial conditions, one has the exact result [18, 19, 20, 21, 22]:

$$
\rho_{n}^{\text {anni }}\left(x_{1}, \ldots, x_{n} ; t\right)=\frac{1}{2^{n}} \rho_{n}^{\text {coal }}\left(x_{1}, \ldots, x_{n} ; t\right) .
$$

The relation (11) has the following simple interpretation: To obtain a configuration of the annihilation process, with the correct weight, select a configuration of the coalescence process but then retain only half of the particles, i.e., randomly, and independently, retain (or discard) each of the original particles with probability 1/2.

This interpretation follows from the well-known observation that the two processes may be realized simultaneously. Starting from a random initial configuration of particles on the line, tag each of the particles with probability $1 / 2$ and run a diffusion-limited coalescence process: When two particles meet they coalesce into a single untagged particle if the parents are alike (either normal or tagged), and into a tagged particle if the parents are different. Clearly, the set of all particles in the system (tagged and untagged) represents a configuration of the coalescence process $A+A \rightarrow A$, while the subset of tagged particles follows the annihilation process $A+A \rightarrow 0$. At any time $t$ a particle is untagged if and only if it has an even number of tagged ancestors at time $t=0$, while the ancestors of two different particles form disjoint sets. It follows that at time $t$ particles are tagged with probability $1 / 2$, independently from one another. 
For the suitable initial conditions for which (11) holds, the density of particles in the anihilation process is half the density in the coalescence process. It is tempting to rescale space in the coalescence process by a factor of two, to impose the same density in both processes. Defining $y_{i}=2 x_{i}$ and effecting the change of variables $\rho_{n}^{\text {coal }}\left(x_{1}, \ldots x_{n} ; t\right) \rightarrow$ $\tilde{\rho}_{n}^{\text {coal }}\left(y_{1}, \ldots y_{n} ; t\right)$, one gets

$$
\rho_{n}^{\text {anni }}\left(x_{1}, \ldots, x_{n} ; t\right)=\tilde{\rho}_{n}^{\text {coal }}\left(y_{1}, \ldots, y_{n} ; t\right) .
$$

This relation has caused some confusion, leading one of us (DbA) to erroneously conclude that since the distribution of particles in coalescence and annihilation are different (as evidenced, for example, from their different inter-particle gap distribution functions) it must be the case that the infinite hierarchy of multiple point correlation functions does not uniquely determine an infinite set of points on the line [22]. Eq. (2) does not mean, however, that the functions $\rho_{n}^{\text {anni }}$ and $\tilde{\rho}_{n}^{\text {coal }}$ are identical: they are applied to different arguments. Indeed, the stretching of space by a factor of two and eliminating half of the particles at random are not generically equivalent 24]. An easy way to see this is by considering the effect of the two operations on a lattice of equally spaced particles: stretching yields a new lattice with double the gap between particles, whereas the random elimination of half the particles leads to a disordered array.

\section{Inter-particle distribution functions (IPDF)}

The hierarchy of multiple-point correlation functions determines the system uniquely. Some quantities, however, such as the IPDF, are notoriously difficult to obtain, using the $\rho_{n}$. This quantity is readily available for the coalescence process, through the method of empty intervals, but not for the annihilation process.

To obtain the IPDF, one needs to compute the probability density $P_{0}\left(x_{1}, x_{2}\right)$ for finding particles at $x_{1}$ and $x_{2}$ and no particles in between. The density probability function of the gap $x$ between particles is then $P_{0}(0, x) / \rho$ [25]. $P_{0}\left(x_{1}, x_{2}\right)$ is, in principle, available from the $\rho_{n}$. Indeed,

$$
P_{0}\left(x_{1}, x_{2}\right)=\rho_{2}\left(x_{1}, x_{2}\right)-\int_{x_{1}}^{x_{2}} d z_{1} \rho_{3}\left(x_{1}, z_{1}, x_{2}\right)+\int_{x_{1}}^{x_{2}} d z_{1} \int_{z_{1}}^{x_{2}} d z_{2} \rho_{4}\left(x_{1}, z_{1}, z_{2}, x_{2}\right)-\ldots
$$

On the right hand side of this equation, events with exactly one particle between $x_{1}$ and $x_{2}$ are counted once by the first term but cancelled by the second term; events with two particles in between are counted once each by the first and third terms, but are cancelled by the second term, which counts these events twice $\left(z_{1}\right.$ representing the first or second intervening particle), etc. In this fashion, only the events with no particles between $x_{1}$ and $x_{2}$ are accounted for at the end.

More generally, one can write down a similar expression for $P_{k}\left(x_{1}, x_{2}\right)$ - the probability density for finding particles at $x_{1}$ and $x_{2}$ and exactly $k$ particles in between:

$$
P_{k}\left(x_{1}, x_{2}\right)=\sum_{n \geq k}(-1)^{n-k}\left(\begin{array}{l}
n \\
k
\end{array}\right) R_{n}\left(x_{1}, x_{2}\right)
$$

where

$$
R_{n}\left(x_{1}, x_{2}\right)=\int_{x_{1}<z_{1}<\cdots<z_{n}<x_{2}} \ldots \int_{2+n}\left(x_{1}, z_{1}, \ldots, z_{n}, x_{2}\right) d z_{1} \cdots d z_{n}
$$

In principle, all the $\rho_{n}$ can be computed explicitly, both in the annihilation and the coalescence process, but their

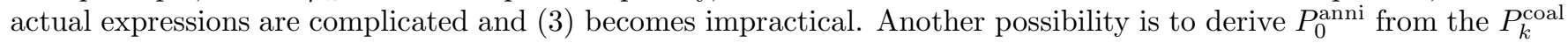
using the correspondence between the configurations of both processes: To obtain a configuration of the anihilation process with particles at positions $x_{1}$ and $x_{2}$ and nothing in between, we start with a configuration of the coalescence process with particles at $x_{1}$ and $x_{2}$ and exactly $k$ other particles in between. Retaining each of these particles with probability $1 / 2$, there is a probability $1 / 4$ that the particles at $x_{1}$ and $x_{2}$ stay put, and a probability $(1 / 2)^{k}$ to kill the $k$ particles in between. Therefore, we have

$$
P_{0}^{\text {anni }}\left(x_{1}, x_{2}\right)=\frac{1}{4} \sum_{k \geq 0} \frac{1}{2^{k}} P_{k}^{\text {coal }}\left(x_{1}, x_{2}\right)
$$

This is but a special case of Eq. (23) in 31], already suggested by Derrida and Zeitak.

Obtaining the $P_{k}^{\text {coal }}\left(x_{1}, x_{2}\right)$ from the $\rho_{n}^{\text {coal }}$, remains still an impractical proposition. Fortunately, in the case of the coalescence process, the method of intervals allows for a more direct way. 


\section{New hierarchy for finding $n$ sequential particles}

Dimension 1 is special in that one can meaningfully discuss the ordering of the particles on the line. Thus, instead of the traditional hierarchy of multiple-point correlation functions, we propose an hierarchy designed to keep track of the sequential order of the particles.

Let $\omega_{n}\left(x_{1}, \ldots, x_{n} ; t\right)$ denote the probability for finding particles at $x_{1}, x_{2}, \ldots, x_{n}$ at time $t$, but no other particles in the intervals $\left(x_{1}, x_{2}\right),\left(x_{2}, x_{3}\right), \ldots,\left(x_{n-1}, x_{n}\right)$. Clearly, the $\omega_{n}$ determine a distribution uniquely. Indeed,

$$
\begin{aligned}
& \rho_{2}\left(x_{1}, x_{2}\right)=\sum_{n=0}^{\infty} \int_{x_{1}<z_{1}<\cdots<z_{n}<x_{2}} \omega_{2+n}\left(x_{1}, z_{1}, \ldots, z_{n}, x_{2}\right) d z_{1} \cdots d z_{n} \\
& \rho_{3}\left(x_{1}, x_{2}, x_{3}\right)=\sum_{k, l} \int_{x_{1}<y_{1}<\cdots<y_{k}<x_{2}<z_{1}<\cdots<z_{l}<x_{3}} \ldots \int_{\substack{x\\
}} \omega_{3+k+l}\left(x_{1}, y_{1}, \ldots, y_{k}, x_{2}, z_{1}, \ldots, z_{l}, x_{3}\right) d y_{1} \cdots d y_{k} d z_{1} \cdots d z_{l},
\end{aligned}
$$

and similar expressions for $\rho_{m}, m>3$. Thus, defining $\omega_{1}(x) \equiv \rho_{1}(x)$, the complete hierarchy of multiple-point correlation functions, $\left\{\rho_{n}\right\}$, can be derived from the hierarchy of sequential particles, $\left\{\omega_{n}\right\}$.

The $\omega_{n}$ are better suited to deal with questions regarding ordered sets of particles. A relevant example are the $P_{k}$, which instead of the infinite sum in (4) are now simply given by

$$
P_{k}\left(x_{1}, x_{2}\right)=\int_{x_{1}<z_{1}<\cdots<z_{k}<x_{2}} \ldots \int_{2+k}\left(x_{1}, z_{1}, \ldots, z_{k}, x_{2}\right) d z_{1} \cdots d z_{k}
$$

and, in particular, $P_{0}\left(x_{1}, x_{2}\right)=\omega_{2}\left(x_{1}, x_{2}\right)$.

In the case of coalescence, the hierarchies $\left\{\rho_{n}\right\}$ and $\left\{\omega_{n}\right\}$ may be derived explicitly through the method of empty intervals. Specifically, the method of intervals yields expressions for $E_{n}\left(x_{1}, y_{1}, \ldots, x_{n}, y_{n} ; t\right)$ - the probability that the intervals $\left(x_{1}, y_{1}\right), \ldots,\left(x_{n}, y_{n}\right)$ be simultaneously empty at time $t$ [30]. The two hierarchies are obtained as spatial derivatives of the $E_{n}$, but evaluated at different points:

$$
\begin{aligned}
\omega_{n}\left(x_{1}, \ldots, x_{n} ; t\right) & =\left.\frac{\partial^{n}}{\partial x_{1} \cdots \partial x_{n}} E_{n}\left(x_{1}, y_{1}, \ldots, x_{n}, y_{n} ; t\right)\right|_{y_{1}=x_{2}, \ldots, y_{n-1}=x_{n}, y_{n}=x_{n}} \\
\rho_{n}\left(x_{1}, \ldots, x_{n} ; t\right) & =\left.\frac{\partial^{n}}{\partial x_{1} \cdots \partial x_{n}} E_{n}\left(x_{1}, y_{1}, \ldots, x_{n}, y_{n} ; t\right)\right|_{y_{1}=x_{1}, \ldots, y_{n}=x_{n}} .
\end{aligned}
$$

Actually, $\omega_{n}$ can be computed somewhat more cheaply, from $E_{n-1}$ rather than $E_{n}$ :

$$
\omega_{n}\left(x_{1}, \ldots, x_{n} ; t\right)=-\left.\frac{\partial^{n}}{\partial x_{1} \cdots \partial x_{n-1} \partial y_{n-1}} E_{n-1}\left(x_{1}, y_{1}, \ldots, x_{n-1}, y_{n-1} ; t\right)\right|_{y_{1}=x_{2}, \ldots, y_{n-1}=x_{n}} .
$$

We have written Eq. (10) merely to showcase the beautiful symmetry between $\rho_{n}$ and $\omega_{n}$.

The first few $\omega_{n}^{\text {coal }}$ computed for coalescence, in the long time asymptotic limit, using the empty intervals derived in [22, 30], are 31]

$$
\begin{aligned}
\omega_{2}^{\text {coal }}\left(x_{1}, x_{2} ; t\right) & =\sqrt{\pi} \rho^{2} \xi_{12} e^{-\xi_{12}^{2}} \\
\omega_{3}^{\text {coal }}\left(x_{1}, x_{2}, x_{3} ; t\right)= & \sqrt{\pi} \rho^{3} \xi_{13}\left(e^{-\xi_{12}^{2}-\xi_{23}^{2}}-e^{-\xi_{13}^{2}}\right) \\
\omega_{4}^{\text {coal }}\left(x_{1}, x_{2}, x_{3}, x_{4} ; t\right)=\quad & \sqrt{\pi} \rho^{4}\left\{\xi_{14}\left(e^{-\xi_{14}^{2}}-e^{-\xi_{14}^{2}-2 \xi_{23}^{2}}+e^{-\xi_{12}^{2}-\xi_{23}^{2}-\xi_{34}^{2}}-e^{-\xi_{12}^{2}-\xi_{24}^{2}}+e^{-\xi_{13}^{2}-\xi_{23}^{2}-\xi_{24}^{2}}-e^{-\xi_{13}^{2}-\xi_{34}^{2}}\right)\right. \\
& \left.+\sqrt{\pi}\left(\xi_{14} \xi_{23} e^{-\xi_{14}^{2}-\xi_{23}^{2}}-\xi_{13} \xi_{24} e^{-\xi_{13}^{2}-\xi_{24}^{2}}+\xi_{12} \xi_{34} e^{-\xi_{12}^{2}-\xi_{34}^{2}}\right) \operatorname{erfc}\left(\xi_{23}\right)\right\}
\end{aligned}
$$

where $\rho=1 / \sqrt{2 \pi D t}$ is the long time asymptotic density of particles in the coalescence process, $\xi_{i j} \equiv\left(x_{j}-x_{i}\right) / \sqrt{8 D t}$, and $\operatorname{erfc}(x)=(2 / \sqrt{\pi}) \int_{x}^{\infty} \exp \left(-u^{2}\right) d u$ is the complementary error function.

Returning to the question of the IPDF in the annihilation process, all the $\omega_{k}^{\text {coal }}$ can be computed, in principle, but obtaining the exact $P_{0}^{\text {anni }}$ from (9) and (6) is not an easy task. Nevertheless, this approach can be used to generate efficient approximations of the IPDF. For instance, for small inter-particle gaps $x=x_{2}-x_{1}$, one need only keep the first few terms in (6), since the probability of finding several particles in the gap becomes negligibly smaller as their numbers increase. Indeed, using just the first term in (6) yields an expression that matches the exact result (Eq (43) in [31]) to order $x^{3}$; the first two terms improve the match up to order $x^{7}$, and three terms up to $x^{12}$. 
However, truncating (6) in this fashion, at any order, gives terrible results for large $x$, as it predicts a Gaussian decay, $\exp \left(-\pi x^{2}\right)$, instead of the correct exponential decay.

For large values of $x$, the simplest assumption that the gaps between particles are independent,

$$
\omega_{2+k}^{\text {coal }}\left(x_{1}, z_{1}, z_{2}, \ldots, z_{k}, x_{2}\right) \approx \frac{\omega_{2}^{\text {coal }}\left(x_{1}, z_{1}\right) \omega_{2}^{\text {coal }}\left(z_{1}, z_{2}\right) \cdots \omega_{2}^{\text {coal }}\left(z_{k}, x_{2}\right)}{\rho^{k}} .
$$

leads to to an exponential decay of the IPDF, $P_{0}^{\mathrm{anni}}(0, x) \simeq 1.6777 \exp (-1.2685 x)$, at large $x$ (where distance is scaled so that the density of particles is equal to 1 ). The same result was already derived in [23], using a similar assumption of uncorrelated particles, and compares favorably with the exact result of $P_{0}^{\operatorname{anni}}(0, x) \sim 1.8167 \exp (-1.3062 x)[31$. Systematic improvements are achieved by taking into account more correlations. For instance, assuming

$$
\omega_{2+l}^{\mathrm{coal}}\left(x_{1}, z_{1}, z_{2}, \ldots, z_{l}, x_{2}\right) \approx \frac{\omega_{3}^{\mathrm{coal}}\left(x_{1}, z_{1}, z_{2}\right) \omega_{3}^{\mathrm{coal}}\left(z_{2}, z_{3}, z_{4}\right) \cdots \omega_{3}^{\mathrm{coal}}\left(z_{l-1}, z_{l}, x_{2}\right)}{\rho^{l}}
$$

(for $l$ odd; for $l$ even, the last term in the product is $\left.\omega_{2}^{\text {coal }}\left(z_{l}, x_{2}\right)\right)$, leads to $P_{0}^{\text {anni }}(0, x) \simeq 1.728976 \exp (-1.285339 x)$.

\section{Discussion}

In summary, we have elucidated the exact relation between diffusion-limited one-species coalescence and annihilation in one dimension. The precise meaning of the similarity between the respective hierarchies of multiple-point correlation functions, Eq. (11), has been clarified: configurations of the annihilation process are obtained by random elimination of half the particles in the coalescence process. This, however, is not equivalent to the stretching of space by a factor of two.

We have also introduced a different hierarchy of probability density functions: the $\omega_{n}\left(x_{1}, \ldots, x_{n} ; t\right)$ — the probability for finding particles at $x_{1}, \ldots, x_{n}$ and no particles in between, at time $t$. This new hierarchy capitalizes on the topological constraints special to one dimension, and is better suited for answering questions concerning specific numbers of particles.

Both the traditional multiple-point correlation functions, the $\rho_{n}$, and the $\omega_{n}$ can be derived from the distribution of empty intervals on the line, in a way that highlights their relationship, Eqs. (10), (11). The two hierarchies determine an infinite system of particles on the line completely, and in particular it is possible to express the $\rho_{n}$ using the $\omega_{n}$, and vice-versa, albeit at the cost of resorting to infinite sums of unwieldy integrals. In this sense, the best situation occurs when the distribution of empty intervals is available, for it yields the $\rho_{n}$ and $\omega_{n}$ directly. This is the case for the coalescence process, but not for annihilation. For annihilation the $\rho_{n}$ are known, but the $\omega_{n}$ are harder to obtain. Derrida and Zeitak have obtained the IPDF (directly related to $\omega_{2}$ ) exactly [31]. Here we presented an alternative approach, based on the relation between the $\omega_{n}$ and $\rho_{n}$, that provides useful approximations.

The situation is not yet completely clear even for coalescence. For example, consider the probability of finding two particles separated by a distance $x$ and having exactly $k$ particles in between, $P_{k}^{\text {coal }}(0, x)$. For small gaps, $x \ll 1 / \rho$, we obtain $P_{k}^{\text {coal }}(0, x) \sim x^{\alpha(k)}$, with $\alpha(k)=1,4,8,13$, for $k=0,1,2,3$, respectively. We have not yet found a satisfactory way to predict $\alpha(k)$ for arbitrary $k$. It remains the subject of future studies.

\section{Acknowledgments}

We are grateful to the organizers of the Dresden 2003 workshop on Non-equilibrium Statistical Physics in Low Dimensions and Reaction-Diffusion Systems for giving us the opportunity to meet and discuss, and to NSF grant PHY-0140094 (DbA) for partial support of this research.

[1] M. Bramson and D. Griffeath, Ann. Prob. 8, 183 (1980).

[2] D. C. Torney and H. M. McConnell, Proc. R. Soc. Lond.A 387, 147 (1983).

[3] Z. Rácz, Phys.Rev. Lett. 55, 1707 (1985).

[4] A. A. Lushnikov, Phys. Lett. A 120, 135(1987).

[5] R. Kopelman, Science 241,1620 (1988); R. Kopelman, S. J. Parus, and J. Prasad, Chem. Phys. 128, 209 (1988).

[6] J. L. Spouge, Phys. Rev. Lett. 60, 871 (1988).

[7] D. J. Balding and N. J. B. Green, Phys. Rev. A 40, 4585 (1989). 
[8] J. G. Amar and F. Family, Phys. Rev. A 41, 3258 (1990); F. Family and J. G. Amar, J. Stat.Phys. 65, 1235 (1991).

[9] P. L. Krapivsky, Physica A198, 150 (1993); 157 (1993).

[10] F. C. Alcaraz, M. Droz, M. Henkel, and V. Rittenberg, it Ann. Phys. 230, 250 (1994).

[11] M. D. Grynberg, T. J. Newman, and R. B. Stinchcombe, Phys. Rev. E 50957 (1994); M. D. Grynberg and R. B. Stinchcombe,Phys. Rev. Lett. 741242 (1995); Phys. Rev. Lett.76 851 (1996).

[12] G. M. Schütz, J. Phys. A 28, 3405 (1995);Phys. Rev. E 53, 1475 (1996).

[13] P. A. Bares and M. Mobilia, Phys. Rev. E 59,1996 (1999); Phys. Rev. Lett. 83, 5214 (1999).

[14] S. Park, J. Park, and D. Kim, Phys. Rev.Lett. 85, 892 (2000); "Two-point correlation functions of the diffusion-limited annihilation in one dimension," cond-mat/0104378 (2001).

[15] S. Habib, K. Lindenberg, G. Lythe, and C. Molina-París, "Diffusion-limited reaction in one dimension: paired and unpaired nucleation," cond-mat/0102270 (2001); S. B. Yuste and Katja Lindenberg, "Subdiffusion-limited reactions," cond-mat/0105338 (2001).

[16] V. Privman, Phys. Rev. E 50, 50(1994).

[17] D. ben-Avraham and S. Havlin, Diffusion andReactions in Fractals and Disordered Systems, (Cambridge University Press, Cambridge, England, 2000).

[18] M. Henkel, E. Orlandini, and G. M. Schütz, J. Phys. A28, 6335 (1995); M. Henkel, E. Orlandini, and J. Santos, Ann. Phys. (NY)259, 163 (1997).

[19] K. Krebs, M. P. Pfanmüller, B. Wehefritz, and H. Hinrichsen, J. Stat. Phys. 78, 1429 (1995).

[20] D. Balboni, P.-A. Rey, and M. Droz, Phys. Rev. E52,6220 (1995); P.-A. Rey and M. Droz, J. Phys. A 30, 1101 (1997).

[21] H. Simon, J. Phys. A 28, 6585 (1995).

[22] T. Masser and D. ben-Avraham, Phys. Rev. E 64, 062101 (2001).

[23] P. A. Alemany and D. ben-Avraham, Phys. Lett. A 206, 18 (1995).

[24] A notable exception is when the particles are distributed randomly, in a Poisson distribution.

[25] Here, and elsewhere in the text, the missing time argument $t$ is implied.

[26] D. ben-Avraham, M. A. Burschka, and C.R. Doering, J. Stat. Phys. 60, 695 (1990).

[27] C. R. Doering, M. A. Burschka, and W. Horsthemke, J. Stat. Phys. 65, 953 (1991).

[28] C. R. Doering, Physica A 188, 386 (1992).

[29] D. ben-Avraham, Mod. Phys. Lett. B 9, 895 (1995).

[30] D. ben-Avraham, Phys. Rev. Lett. 81, 4756 (1998).

[31] B. Derrida and R. Zeitak, Phys. Rev. E 54, 2513 (1996).

[32] M. Khorrami and A. Aghamohammadi, Phys. Rev. E63, 042102 (2001); M. Alimohammadi, M. Khorrami, and A. Aghamohammadi, "Exactly solvable models through the empty interval method," cond-mat/0105124 